\title{
Strain Estimation in a Glass Beam using Operational Modal Analysis
}

\author{
Manuel L. Aenlle ${ }^{1}$, Anders Skafte ${ }^{2}$, Pelayo Fernández ${ }^{1}$ and Rune Brincker ${ }^{2}$. \\ ${ }^{1}$ University of Oviedo. Department of Construction and Manufacturing Engineering. Campus de \\ Gijón s/n. Ed. Oeste. Bloque 7. 33203 Gijón. Spain. \\ ${ }^{2}$ Department of Engineering, Aarhus University, Edison Building, Finlandsgade 22, 8200 Aarhus \\ $\mathrm{N}$, Denmark.
}

\begin{abstract}
A potential application of operational modal analysis is the prediction of strain or stress time histories which, on the other hand, are one of the most important sources of uncertainty in fatigue design and remaining fatigue life calculations. This is due to the difficulty of estimating the stiffness, mass and damping properties with accuracy, as well as the use of simplified loading models.

In this work, it is proposed a methodology to estimate the strain or stress time histories at any point of a beam, which need the strain mode shapes, expanded using a finite element model and the modal coordinates of the responses, which contain the information corresponding to the damping and the natural frequencies. Experimental tests were carried out on a monolithic glass beam to study the accuracy obtained in the strain mode shapes.
\end{abstract}

Keywords: Operational Modal Analysis, Strains, Strain Mode Shapes, Modal Assurance Criterion, Glass Elements.

\section{INTRODUCTION}

Operational modal analysis (OMA) is a technique with many potential applications in civil structures $[1,2]$ and mechanical systems [3]. OMA makes use of the natural or operating loads to excite the structure and the modal tests can be performed with the structure in operation subject to natural or operational loads [4].

Experimental modal analysis has been widely used in the past to validate finite element models, model updating, health monitoring, structural modification, damage detection, etc. In this paper it is proposed a methodology to estimate strains or stresses in any arbitrary point of the structure, which combines a numerical model, previously updated using the modal parameters identified by OMA and the acceleration time histories recorded at several points of the structure. With this methodology, the only information needed from the finite element model is the strain mode shapes whereas the information corresponding to natural frequencies and damping are contained in the modal coordinates which are estimated from the experimental responses [5, 6].

This paper only deals with the estimation of the strain mode shapes. The accuracy in the estimation of strain mode shapes has been studied by experimental tests carried out on a monolithic glass beam. Several accelerometers were used to measure the responses whereas some strain gauges were attached to identify the strain mode shapes by operational modal analysis. The strain mode shapes from the strain gauges are compared with those derived from the mode shapes identified from the accelerations and expanded using a finite element model.

\section{THEORY}

The methodology proposed in this paper is particularized to a plane Euler-Bernoulli beam. 
For an Euler-Bernoulli beam (see Figure 1) the bending moment and the curvature are related by the equation:

$$
E I_{z} \frac{d^{2} y}{d^{2} x}=M_{z}
$$

where $\mathrm{E}$ is the Young's modulus, $\mathrm{I}_{\mathrm{z}}$ is the second moment of the cross section about $\mathrm{z}$ axis, $\mathrm{y}$ is the vertical displacement and $\mathrm{M}_{\mathrm{z}}$ is the bending moment.

The stress can be calculated by means of the Navier's Law equation, i.e:

$$
\sigma(x)=\frac{M_{z}}{I_{z}} \cdot h
$$

where $\mathrm{h}$ is the beam thickness.

If Eqs. (1) and (2) are combined, a relation between the strain and the curvature is obtained by:

$$
\varepsilon(x)=h \cdot \frac{d^{2} y}{d^{2} x}
$$

On the other hand, if a finite element model is used, the displacement in any arbitrary point of an element (see Figure 2) is given by [7]:

$$
y(x)=N_{e} \cdot u_{e}
$$

where $\mathrm{N}_{\mathrm{e}}(\mathrm{x})$ and $\mathrm{u}_{\mathrm{e}}$ are vectors containing the shape functions and the nodal displacements, respectively.

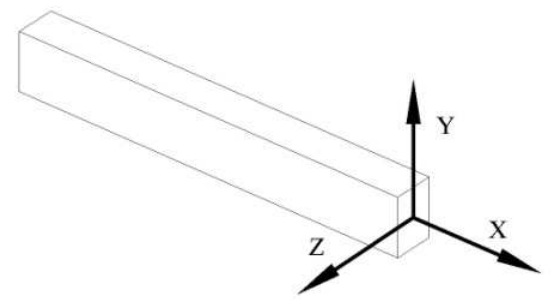

Fig. 1 Example of Euler-Bernoulli beam

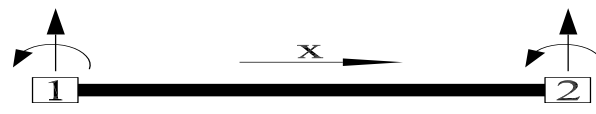

Fig. 2 Linear beam element

If Eq. (4) is combined with Eq. (3), the following expression is inferred:

$$
\varepsilon(x, t)=N_{e} "(x) \cdot u_{e}(t) h
$$

Using model-superposition [8], the vector $\mathrm{u}_{\mathrm{e}}(\mathrm{t})$, corresponding to each element, can be expressed in terms of mode shapes, $\Phi_{\mathrm{e}}$, and the modal coordinates, $q(t)$, as follows $[5,6]$ :

$$
u_{e}(t)=\phi_{e} \cdot q(t)
$$

where $\phi_{e}$ is a matrix containing the components of mode shapes at the DOF's of the element.

Finally, if Eq. 6 is substituted in Eq. 5, the strains at any point of the beam element can be calculated by means of the expression:

$$
\varepsilon(x, t)=N_{e} "(x) \cdot \phi_{e} \cdot q(t) \cdot h
$$

In Eq. (7) two main terms can be identified: the modal coordinates, $q(t)$, and the strain mode shapes:

$$
\phi_{\varepsilon}(x)=\phi_{e}^{\prime \prime}(x)=N_{e}(x)^{\prime \prime} \cdot \phi_{e}
$$

which are estimated using the shape functions and the components of the updated mode shapes at the DOF's of the element. 


\subsection{Steps}

\section{Finite Element Model}

The first stage of the proposed method is to assemble a numerical model [7]. This step can be avoided if the mode shapes can be fitted using analytical functions, splines, etc.

\section{Modal Parameters}

A modal identification is performed to obtain the natural frequencies, $\mathrm{f}_{\text {exp }}$, and mode shapes, $\Phi_{\text {exp, }}$ of the structure. The identification may be performed by e.g. Stochastic Subspace Identification (SSI) [9], or Frequency Domain Decomposition (FDD) [10]. The FDD is used in this paper as implemented in the ARTeMIS Extractor software. The FDD is based on calculation of Spectral Density Matrices of the measured data series by discrete Fourier transformation. For each frequency line the Spectral Density Matrix is decomposed into auto spectral functions corresponding to a single degree of freedom system (SDOF) [10].

\section{Modal Scaling}

An important disadvantage of OMA is that not all modal parameters can be estimated [5, 11]. Since the forces are unknown, the mode shapes can not be mass normalized and only the un-scaled mode shapes can be obtained for each mode. However, the procedure proposed in this paper can be used with both mass normalized or arbitrary scaled mode shapes. If mass normalized mode shapes are used, the scaling factors can be estimated by the mass change method [5, 11] which consists of modifying the dynamic behaviour of the structure adding masses in the points of the structure where the mode shapes are known and performing repeated modal tests on both the original and the perturbed structure

\section{Model Up-dating and Modal Expansion of the Mode Shapes}

In this stage, the finite element model is updated using the experimental modal parameters estimated with OMA [12]. After updating, a transformation matrix $\mathrm{T}$ is obtained from:

$$
\phi_{\exp }^{m}=\phi_{F E}^{m} \cdot T
$$

where subscripts 'exp' and 'FE' indicates experimental and numerical mode shapes, respectively, and superscript ' $\mathrm{m}$ ' indicates measured DOF's. In expression (9), the experimental mode shapes are assumed to be a linear combination of the numerical mode shapes [12]. Then, the experimental mode shapes are expanded to the unmeasured degrees of freedom by the expression:

$$
\phi_{e x p}^{u m}=\phi_{F E}^{u m} \cdot T
$$

where superscript ' 'um' indicates unmeasured DOF's. The same procedure has to be followed in case of un-scaled mode shapes.

\section{Modal Coordinates}

The experimental displacement modal coordinates, $q_{\exp }(t)$, have to be calculated from the measured acceleration, $\ddot{u}_{\text {exp }}(t)$. The acceleration modal coordinates, $\ddot{q}_{\text {exp }}(t)$, are firstly obtained by means of the expression:

$$
\ddot{q}_{\text {exp }}(t)=\phi_{\text {exp }}^{-1} \cdot \ddot{u}_{\text {exp }}(t)
$$

where $\ddot{u}_{\exp }(\mathrm{t})$ is the measured acceleration and $\phi_{\text {exp }}$ is the experimental mode shape matrix. Then, the displacement coordinates, $q(t)$, are estimated by a double integration of $\ddot{q}_{\text {exp }}(t)$. In this work a double integration in the frequency domain was used as follows:

$$
q_{\exp }(\omega)=-\frac{\ddot{q}_{\exp }(\omega)}{\omega^{2}}
$$

and the corresponding modal coordinates in the time domain can be obtained by inverse Fourier Transform.

If un-scaled mode shapes, $\psi$, are used, the scaling factors are needed. The mass normalized, $\phi$, and the un-scaled mode shapes, $\psi$, are related by:

$$
\phi=\psi \cdot \alpha
$$

where $\alpha$ is a diagonal matrix containing the scaling factors. 
If Eq. (13) is substituted in Eq. (6), it results in:

$$
u_{e}(t)=\psi_{e} \cdot \alpha \cdot q(t)
$$

or, alternatively:

$$
u_{e}(t)=\psi_{e} \cdot q^{*}(t)
$$

where $q^{*}(t)$ is denoted here as pseudo modal coordinate, which is estimated by means of the expression:

$$
\ddot{q}_{\text {exp }}^{*}(t)=\psi_{\text {exp }}^{-1} \cdot \ddot{u}_{\text {exp }}(t)
$$

\section{Strain Estimation}

Finally, combining equations (5), (6) and (11), the strains can be determined by the equation:

$$
\varepsilon(x, t)=N_{e} "(x) \cdot \phi_{e} \cdot q_{\text {exp }}(t) \cdot h
$$

For the case of mass normalized mode shapes. In the case of un-scaled mode shapes, equations (5), (15) and (16) give:

$$
\varepsilon(x, t)=N_{e} "(x) \cdot \psi_{e} \cdot q_{\text {exp }}^{*}(t) \cdot h
$$

\section{EXPERIMENTAL TESTS}

A monolithic glass beam was used to perform the tests (see Figure 3). The beam was $1 \mathrm{~m}$ long, showing a $100 \mathrm{x} 10 \mathrm{~mm}$ rectangular section. Two support configurations were considered in the investigation: simply supported (SS) and simply supported with a cantilever (SSC). A finite element model was assembled in ABAQUS to obtain a set of mode shapes, using linear beam elements (figure 2).

\subsection{Operational modal analysis}

The beam was excited applying hits with a small piece of wood along the beam trying to reproduce a stationary broad banded load. The responses were measured using accelerometers with a sensitivity of $100 \mathrm{mv} / \mathrm{g}$, weighing 5 grams and located as shown in Figure 3. The tests were carried out using a sampling frequency of $2000 \mathrm{~Hz}$ during a period of approximately 4 minutes. Seven $350 \Omega$ strain gauges were also attached (figure 3 ) to obtain the strain mode shapes by OMA.

The modal parameters were identified by Enhanced Frequency Domain Decomposition (EFDD) [10]. The normalized singular values are presented in Figures 4 and 5. The first five natural frequencies, for both support configurations, are shown in Table 1.

Table 1 Natural frequencies [Hz]

\begin{tabular}{lcccccc}
\hline \multicolumn{2}{l}{ MODE } & 1 & 2 & 3 & 4 & 5 \\
\hline SS & Accelerometers & 25.22 & 96 & 214.7 & 382.6 & 593.2 \\
& Strain Gages & 25.23 & 96 & 214.7 & 382.7 & 592.3 \\
\hline SSC & Accelerometers & 36.1 & 104.4 & 187.9 & 368.5 & 620.7 \\
& Strain Gages & 36.1 & 104.3 & 187.9 & 368.5 & 621.9 \\
\hline
\end{tabular}


The Modal Assurance Criterion (MAC) between the numerical and the experimental modes is presented in table 2.
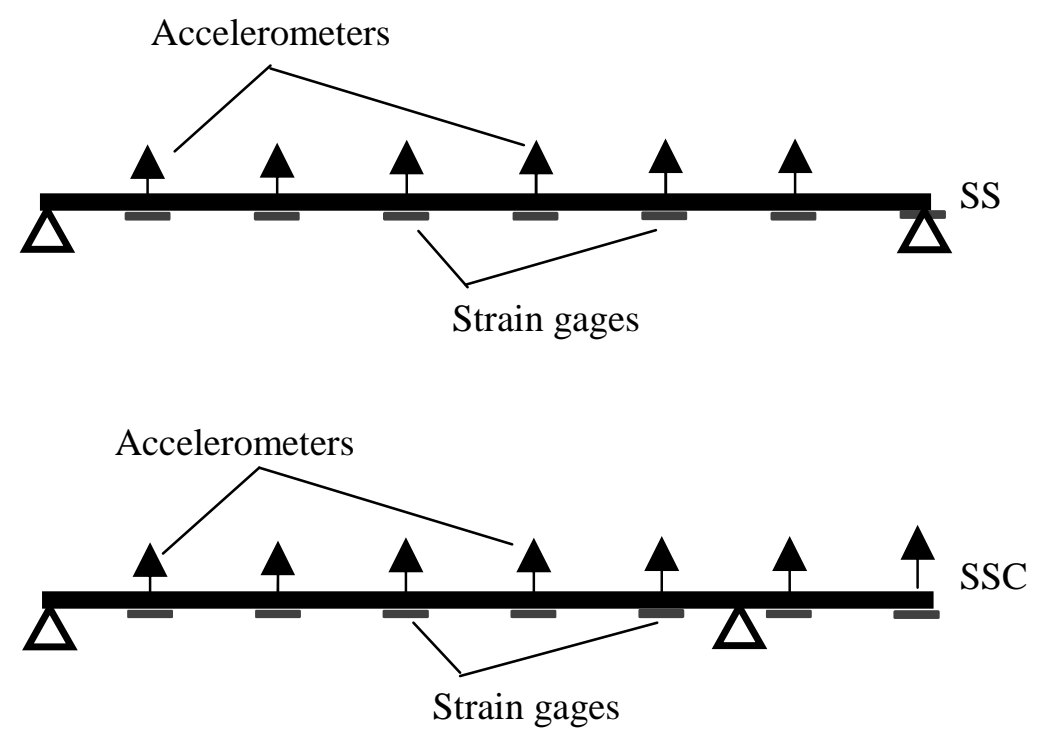

Fig. 3 Accelerometers and strain gage configuration

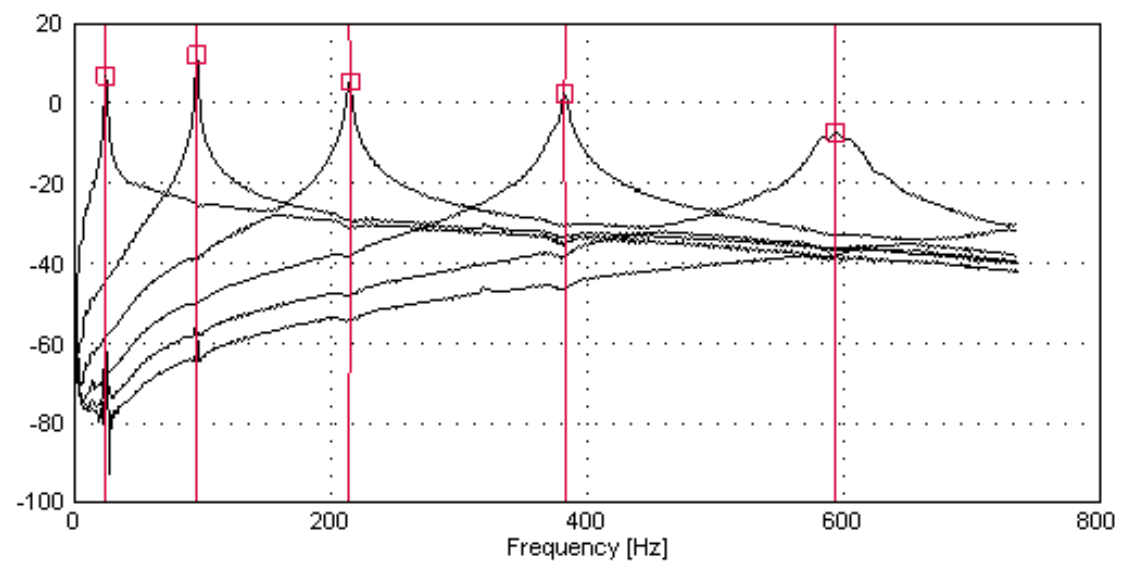

Fig. 4 Modes identified from the accelerometers in SS configuration by EFDD

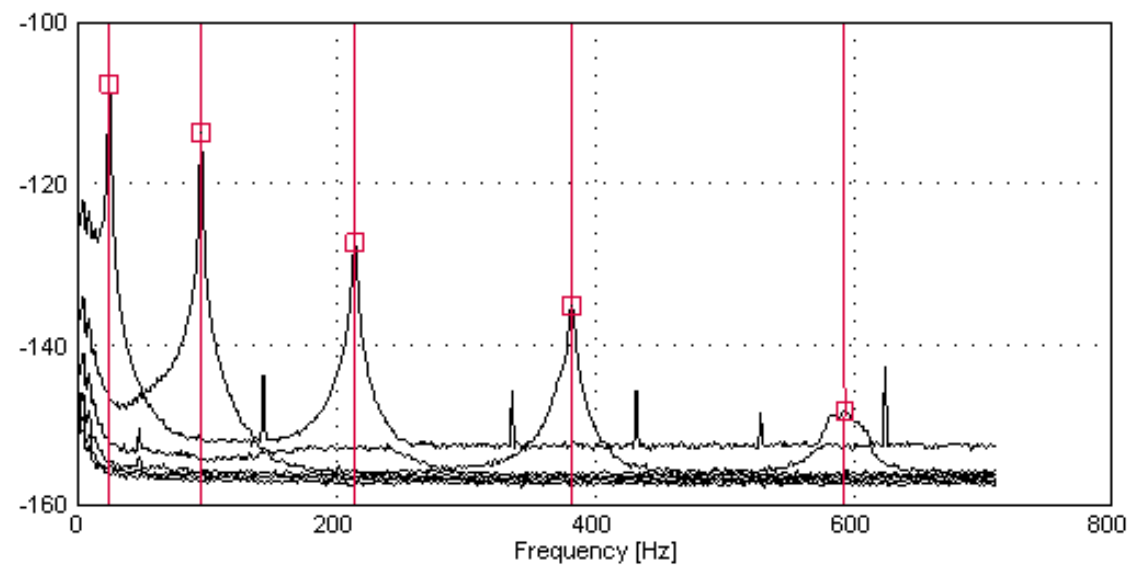

Fig. 5 Modes identified from the strain gages in SS configuration by EFDD 
Table 2 MAC between the numerical and the experimental models

\begin{tabular}{ccccccc}
\hline & \multicolumn{3}{c}{ SS (Accelerometers) } & \multicolumn{2}{c}{ SSC (Accelerometers) } \\
\hline \multirow{2}{*}{ Numerical } & 0.9998 & 0.0000 & 0.0001 & 0.9980 & 0.0561 & 0.0207 \\
\multirow{2}{*}{ Model } & 0.0001 & 0.9994 & 0.0001 & 0.0754 & 0.9990 & 0.0941 \\
& 0.0002 & 0.0003 & 0.9986 & 0.0569 & 0.1294 & 0.9831 \\
\hline
\end{tabular}

\subsection{Results}

In figure 6 are presented the first three strain mode shapes of the simply supported configuration (SS) obtained using Eqs. (9) and (10), together with those obtained by modal identification of the strain gauges. The finite element model was not updated as there was a good agreement between the experimental and the numerical mode shapes (see table 2). It can be observed that there is a good agreement between the results provided by both methods, except at the borders of the beam.

If linear beam elements $(\mathrm{C} 1)$ are used in the finite element model, the continuity in the deflection and the rotation is guaranteed. However, there is a lack of continuity in the curvature across inter-element boundaries. In this work, the curvature has been smoothed at each node averaging the curvatures corresponding to each element connected at the node. However, this technique cannot be applied at the borders of the beam, reason by which the largest error appears in these positions. In table 2 is presented the MAC between the strain mode shapes obtained by both methods. If the strain mode shapes components at the borders are not considered, a good agreement is achieved (see table 3) and the MAC improves significantly.

In figure 7 the results for the simply supported with cantilever configuration (SSC) are presented. Again, a good agreement exists between the results of both methods. With respect to the MAC, presented in tables 3 and 4 , the same conclusions as for the SS configuration can be established.

Table 3 MAC in the strain mode shapes

\begin{tabular}{cccccccc}
\hline & \multicolumn{2}{c}{ SS (Accelerometers) } & \multicolumn{3}{c}{ SSC (Accelerometers) } \\
\hline Strain Gauges & 0.9827 & 0.0007 & 0.0079 & 0.9478 & 0.0001 & 0.0163 \\
& & & & & 0.0002 & 0.9757 & 0.0072 \\
& 0.0002 & 0.9507 & 0.0011 & 0.0005 & 0.0044 & 0.9831 \\
\hline
\end{tabular}

Table 4 MAC in the strain mode shapes when border points are not considered

\begin{tabular}{lccccccc}
\hline & \multicolumn{2}{c}{ SS (Accelerometers) } & \multicolumn{3}{c}{ SSC (Accelerometers) } \\
\hline Strain Gauges & 0.9902 & 0.0007 & 0.0079 & 0.9822 & 0.0001 & 0.0164 \\
& 0.0002 & 0.9997 & 0.0011 & 0.0002 & 0.9995 & 0.0071 \\
& & & & & 0.0005 & 0.0044 & 0.9967 \\
\hline
\end{tabular}



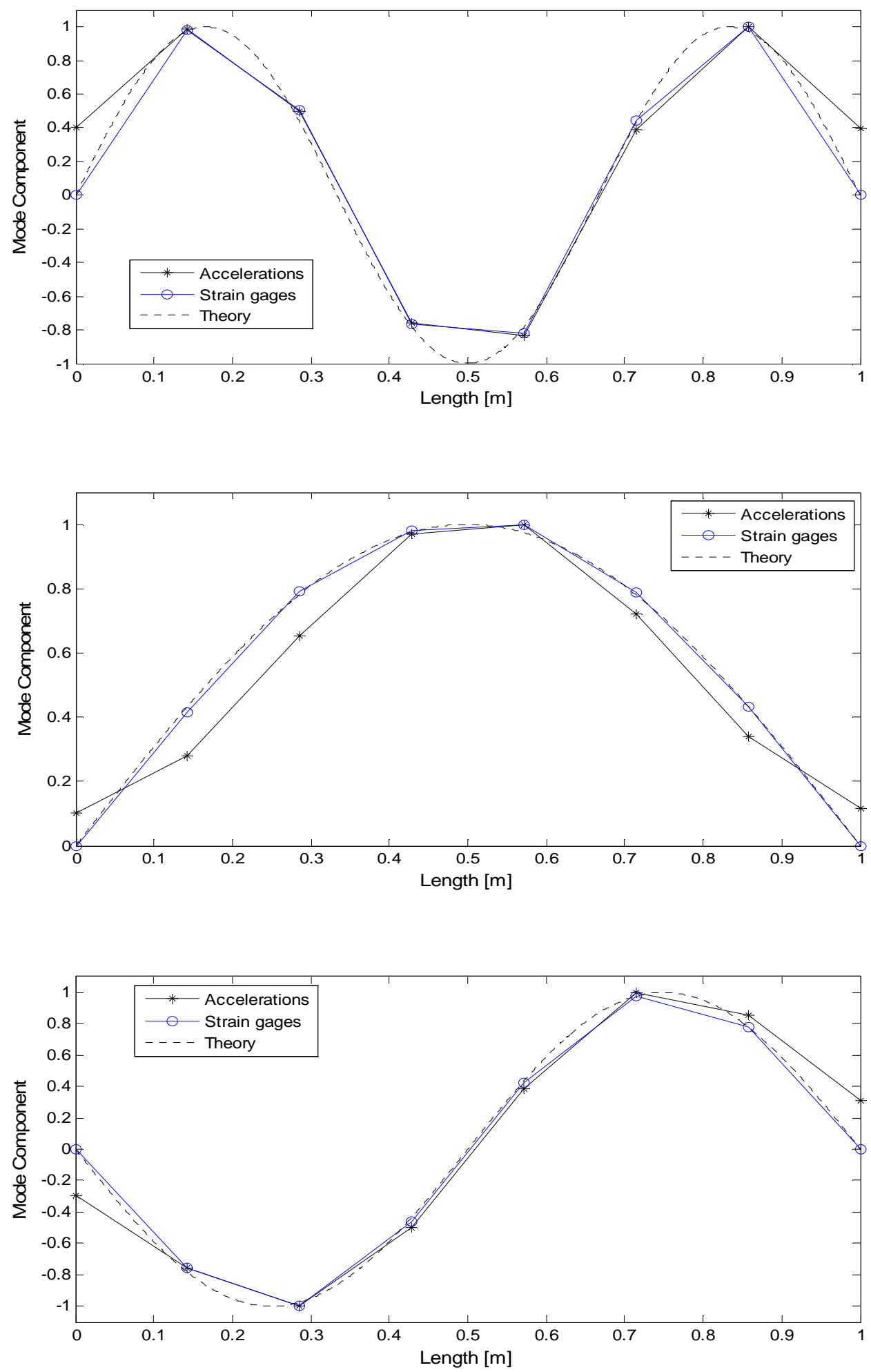

Fig. 6 Strain mode shapes for the first three modes in SS configuration 

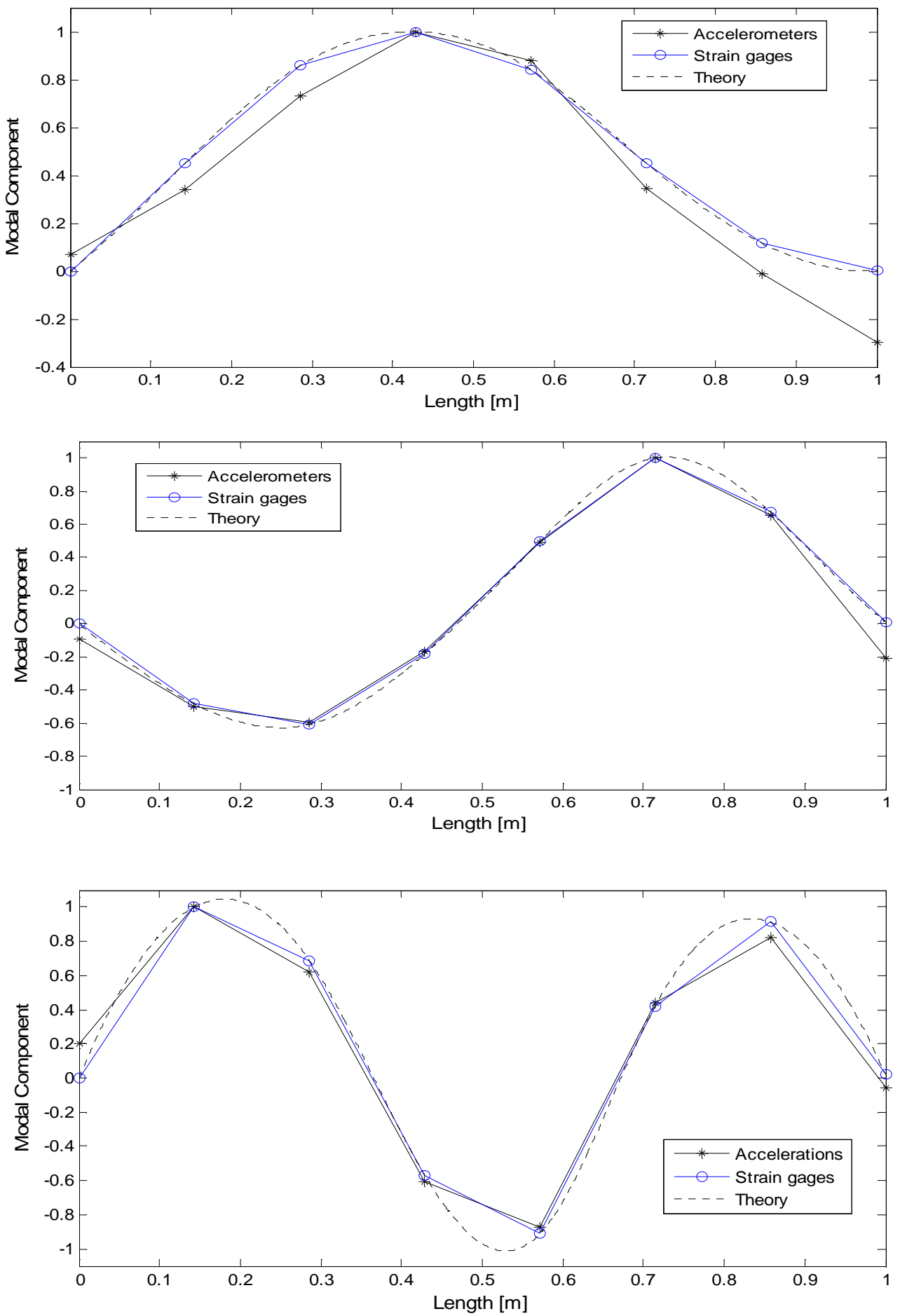

Fig. 7 Strain mode shapes for the first three modes in SSC configuration 


\section{CONSLUSIONS}

- A methodology to estimate strains at any arbitrary point of a structure, which combines a numerical model, previously updated using the modal parameters identified by OMA, and the acceleration time histories recorded at several points of the structure, is proposed. The methodology only needs the strain mode shapes and the modal coordinates estimated from the experimental responses.

- The accuracy obtained with this technique in the strain mode shapes has been investigated performing experimental tests on a monolithic glass beam using different support configurations. The results show the difficulty of estimating the strain mode shapes at the borders of the beam with accuracy. However, a good precision has been obtained for all the modes in the rest of the points of the beam.

\section{ACKNOWLEDGEMENTS}

The economic support given by the Spanish Ministry of Science and Innovation through the project BIA 2011-28380C02-01 is gratefully appreciated.

\section{REFERENCES}

[1] Cantieni, R. Experimental Methods Used in System Identification of Civil Engineering Structures. In Proceedings of the International Operational Modal Analysis Conference (IOMAC), Copenhagen, pp. 249-260, 2005.

[2] Cunha, A. and Caetano, E. From Input-Output to Output-Only Modal Identification of Civil Engineering. In Proceedings of the International Operational Modal Analysis Conference (IOMAC), Copenhagen, pp. 11-27, 2005.

[3] Møller, N. and Brincker, R. and Herlufsen, H. and Andersen P. Modal Testing Of Mechanical Structures Subject To Operational Excitation Forces. In:Proceedings of The 19th International Modal Analysis Conference (IMAC). Kissimmee, Florida, pp.262-269, 2001.

[4] Brincker, R., Ventura, C and Andersen, P. Why output-Only Modal Testing is a Desirable Tool for a Wide Range of Practical Applications. In Proc. of the International Modal Analysis Conference (IMAC) XXI, paper 265, 2003.

[5] Pelayo, F. Scaling Factors and Straines Estimation in Structures by means of Operational Modal Analysis. Ph.D. University of Oviedo, 2010.

[6] Pelayo Fernández, Manuel López Aenlle, Rune Brincker and Alfonso Fernández-Canteli. Stress estimation in structures using operational modal analysis. 3th International Operational Modal Analysis Conference (IOMAC). Portonovo (Ancona), Italy, 2009.

[7] Zienkiewicz, O.C. and Taylor, R.L. The Finite Element Method for Solid and Structural Mechanics $\left(6^{\text {th }}\right.$ edition). Eselvier. UK, 2005.

[8] Clough, Ray W. and Penzien, J. Dynamics of structures, 2nd edition, McGraw-Hill, New York, 1993.

[9] Van Overschee, P. and De Moor, B. Subspace identification for linear systems: Theory, implementation, applications. Kluwer Academic Publishers, 1996.

[10] Brincker, R., Zhang, L and Andersen, P. Output-Only Modal Analysis by Frequency Domain Decomposition. Smart Materials and Structures, 10, pp. 441-445, 2001.

[11] Manuel López-Aenlle, Pelayo Fernández, Rune Brincker and Alfonso Fernández-Canteli. Scaling-factor estimation using an optimized mass-change strategy. Mechanical Systems and Signal Processing, (24) 1260-1273, 2010.

[12] Friswell, M.I. and Mottershead, J.E. Finite Element Model Updating in Structural Dynamics. Kluwer Academic Publishers, 1995. 